\section{La formation continue en cabinet médical}

C'est l'évidence pour tout le monde. On apprend le métier de plombier, de boulanger ou de maçon chez un plombier, un boulanger ou un maçon. Et on apprend celui de médecin de premier recours chez un médecin de premier recours. C'est un fait reconnu par la LAMal, qui prescrit qu'une partie de la formation postgraduée des médecins doive se dérouler dans le secteur des soins ambulatoires.

C'est donc clair?

Un projet pilote sur la formation des médecins de premier recours en cabinet médical (assistanat) est conduit sur trois ans par le Collège de Médecine de premier recours avec un grand succès. Vous trouverez dans ce numéro deux articles à ce sujet, décrivant le déroulement du projet et évaluant ses résultats [1, 2]. Tout le monde est enthousiaste.

Alors, tout va bien?

Dans les directives européennes sur la formation postgraduée en médecine de premier recours, l'assistanat en cabinet médical est bien ancré. Au Canada, en Australie et aux Etats-Unis, c'est un fait établi.

Alors, tout va bien?

Tout va bien? Eh bien non!!!!

La logistique du projet est en élaboration. Il ne se passe pratiquement pas de semaine sans que de nouveaux cabinets médicaux soient habilités à la formation, l'administration est optimisée en permanence, tout est en bonne voie.

Le projet est actuellement financé par les médecins-assistants qui renoncent $\dot{a}$ une partie de leur salaire et par des contributions des maîtres de stage, de la FMH, de l'ASMAC et des sociétés de Médecine de premier recours. Cet engagement financier restera indispensable à l'avenir.

Malheureusement, le problème du financement de l'assistanat en cabinet médical pour TOUS les futurs médecins de premier recours lou mieux en- core pour tous les médecins pratiquant en cabinet) n'est pas encore résolu.

La loi prévoit le paiement de la formation postgraduée des médecins par les assistants. Ceci est possible dans les hôpitaux, car les médecinsassistants y travaillent pendant leur formation. Mais, ainsi que l'évaluation du projet pilote le montre, ce n'est pas réalisable en cabinet médical, car la présence d'un assistant ne conduit pas en général à un accroissement des contacts avec les patients et constitue plutôt une charge supplémentaire pour le maître de stage.

Cependant, comme l'étude le démontre clairement, les futurs médecins de premier recours se sentent beaucoup mieux préparés à leurs activités futures lorsqu'ils ont effectué un stage d'assistanat en cabinet médical. Tous les intervenants concernés du secteur de la santé publique devraient donc avoir le plus grand intérêt à faire profiter à l'avenir tous les médecins praticiens de cette préparation professionnelle, que la loi prescrit d'ailleurs - comme il est dit plus haut.

Ou bien est-ce qu'ici aussi la qualité ne doit rien coûter?

En tout cas, je recommande vivement la lecture de ces deux articles à tous les politiciens, directeurs de départements sanitaires, responsables de caisse maladie, directeurs financiers d'entreprises pharmaceutiques, et à tous ceux qui restent encore sceptiques. Un investissement dans l'amélioration de la qualité de notre médecine de premier recours en vaut la peine, c'est sûr!

Dr Marc Müller,

Président du Collège de Médecine de premier recours

1 Rindlisbacher R, Battaglia M. Formation postgraduée au cabinet du médecin de premier recours. Organisation et mise en application du projet pilote de trois ans $1^{\mathrm{er}}$ juillet 1998-30 juin 2001. Bull Med Suisses 2002;83(14):643-5.

2 Schläppi P, Hofer D, Bloch R. La forme d'apprentissage "assistanat au cabinet médical" a fait ses preuves. Bull Med Suisses 2002;83(14):646-53. 